\title{
Implementation of Knowba Filtering Method for Intereference Rejection in NBDSSS
}

\author{
Rekha R S, K. N. Muralidhar
}

\begin{abstract}
Spread spectrum communication is a communication method that deliberately makes the bandwidth of the transmitted waveform larger than would be required to transmit the data over the channel.One of the most encouraging multiplexing techniques for present and forthcoming telecommunications services, such as private communications, ad-hoc wireless communications, third-generation cellular telephony and sensor networks, is the CDMA(code division multiple access) implemented with direct-sequence (DS) signaling. DS-CDMA's benefits include superior operation in multi-path settings, flexibility in channel allocation, enhanced capacity in fading settings, and the capability to share bandwidth with narrowband communication technologies without deteriorating the efficiency of either system. In our work,we are proposing a new know-ba strategy to dismissing interference where originally monitoring signal environment with high precision is called from the library and subsequently based on data that is appropriate for dismissing interference. Various interference scenarios are simulated using computer simulations to show the efficiency of the method.
\end{abstract}

Keywords : DS-CDMA, sensor networks, interference, knowledge based strategy.

\section{INTRODUCTION}

Higher and greater information requirements and communication system specifications are being suggested with the growth of contemporary society[1]-[6 ]. However, it is hard and sometimes even impossible to meet these needs and specifications with standard modulation and coding schemes[7]. Therefore, in reaction to these circumstances and requests, some more sophisticated transmission methods using Orthogonal Frequency-Division Multiplexing (OFDM) are increasing [ 8]-[10 ]. Spread spectrum signaling, other than OFDM, is one of the most extensively used communications techniques.Specifically, in comparison with its data rate[7 ], it is a signaling system that uses a very large transmission bandwidths. Bandwidth sacrifice[11] is a trade-off for reduced transmitter energy, privacy, and security. Therefore, Code Division Multiple Access (CDMA) is the most frequently implemented signaling of the spread spectrum[12 ]. All these above-mentioned merits and characteristics can not be accomplished through standard transmission methods and therefore the spreading of spectrum signaling maintains a significant place in modern

Revised Manuscript Received on December 30, 2019.

* Correspondence Author

Rekha R S, Department of Electronics and Communication, Government Engineering College, Chamarajanagara, Karnataka, India

K. N. Muralidhar, Department of Electronics and Communication, Adichunchanagiri University, Karnataka, India

(C) The Authors. Published by Blue Eyes Intelligence Engineering and Sciences Publication (BEIESP). This is an open access article under the CC BY-NC-ND license (http://creativecommons.org/licenses/by-nc-nd/4.0/) communication engineering research[12]-[15 ]. Spread spectrum communication is a technique of communicating whereby the bandwidth of the transmitted waveform is purposely made broader than would be necessary to communicate the data over the channel. Spread communication with spectrum has excellent anti-noise and anti-multipath fading capabilities. It can efficiently decrease deliberate and unintentional interference. The spreading is accomplished in one of two possible manners

i) In Direct sequence spread spectrum, there is data bits, superimposed upon wide bandwidth spreading sequence. This sequence is frequently produced from a linear feedback shift register and is often called pseudorandom noise sequence.

ii) Frequency hopping: The spectrum is spread by taking the original signal and periodically changing its carrier frequency in a seemingly random manner. Interference rejection schemes are very much essential for smooth functioning of systems.

\section{ESSENTIAL CONCEPTS AND ADVANTAGES OF SPREAD SPECTRUM SIGNALING}

This Spread spectrum signals are defined by the fact that the signals with the necessary bandwidth $\mathrm{W}$ are much bigger than the information rate $\mathrm{R}[7$ ]. To evaluate this feature, we can identify the bandwidth expansion[7 ].

$\mathrm{B}_{\mathrm{e}}=\mathrm{W} / \mathrm{R}=\mathrm{T}_{\mathrm{b}} / \mathrm{T}_{\mathrm{c}}$----- 1

Usually, $B_{e}=1$, should be an integer for practical applications for spreading spectrum signals[7 ]. As we learned from the review of literature[17]-[18 ], we understand that bandwidth is very valuable and could a huge amount of dollars[19 ]. Therefore, it is needed to explore why it is worth implementing such a bandwidth-efficient method and how it can be used to enhance a communication system's efficiency. In short, the immunity against a multitude of interference can be considerably enhanced by using a big quantity of bandwidth and high consistency is achieved[6]. This is essential for certain unique channels of communication used for rescue, army and other crises[20]-[21]. Also, as the bandwidth is sufficiently big, it is possible to reduce the respective transmitter power, which is more energy-efficient and suitable to be enforced for some unique occasions when a rigorous transmitter energy threshold is set[23 ]. In addition, the safety may also be improved along with the low power characteristic, since the truncation of the spread spectrum signals acts as noise for the band-limited receivers and cannot be intentionally intercepted and blocked without the prior information about the spread spectrum signaling scheme. This type of signal is therefore categorized as a signal with low probability of interception (LPI)[24 ]. 


\section{Implementation of Knowba Filtering Method for Intereference Rejection in NBDSSS}

The principle of immunity from interference with inter-symbols (ISI) brought about by signaling the spread spectrum. Although various transmitters share the common bandwidth, they encode their data using distinct coding systems and so only the expected receivers can decode the respective received signals. Therefore, indistinguishable and discarded signals coded by some other codes[25 ]. The ISI can thus be reduced. Since the codes used to distribute sequences are a sequence of distinct pseudorandom patterns, this method is called Code Divison Multiple Access, the most frequently used spread spectrum signaling technique[26 ].

\section{KNOWBA (KNOWLEDGE BASED) FILTER DESIGN[27]}

This section provides simulation outcomes for two distinct situations to show the performance improvement obtained by our knowledge-based cancellation of interference over the adaptive transversal filter. In terms of bit error rate (BER), performance is defined. For perfect synchronization, the BER curves were obtained. Different combinations of jamming signals are used in the two situations where each jammer's strength alone is of a magnitude big enough to override the spread-spectrum system's processing gain, resulting in important demodulator output mistakes.

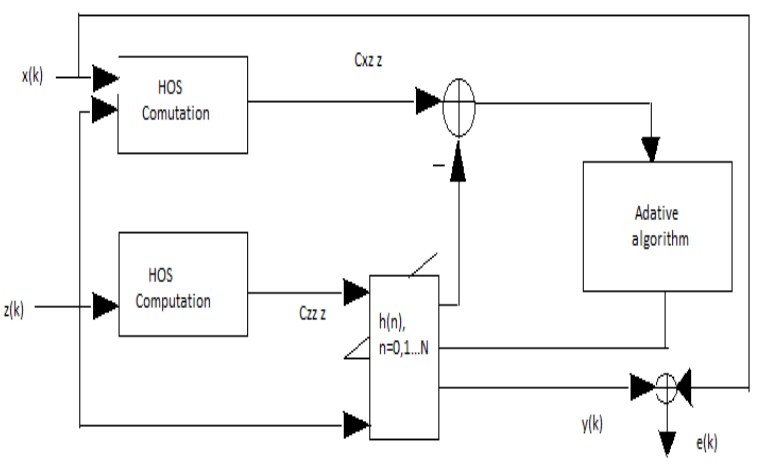

Fig 1 :Block diagram adaptive knowledge based filter

Use gradient and genetic algorithm to use the block diagram for HOS (Higher Order Statistics). Let $\{x(k))$ and $\{\mathrm{z}(\mathrm{k})$ ) denote the sequence of the received signal and the sequence of the interference signal, both of which are divided into samples in chip rate.

$\mathrm{x}(\mathrm{q})=\mathrm{s}(\mathrm{q})+\mathrm{I}(\mathrm{q})+\mathrm{n}_{\mathrm{p}}(\mathrm{q})$

$\mathrm{z}(\mathrm{q})=\mathrm{w}(\mathrm{q})+\mathrm{n}_{\mathrm{r}}(\mathrm{q})$

where $\{s(q))$ is DS signal, $\{\mathrm{I}(\mathrm{q}))$ is narrow band interference signal, $\{w(q)\}$ is a zero-mean, non-Gaussian reference signal highly correlated with the interference.

Let $\mathrm{C}_{\mathrm{xzz} \ldots \mathrm{z}}\left(\mathrm{m}_{1}, \mathrm{~m}_{2}, \ldots \ldots \mathrm{m}_{\mathrm{n}-1}\right)$ means the nth order crossculmulants between the reference and received signal, $\mathrm{C}_{\mathrm{yzz} \ldots . . \mathrm{z}}\left(\mathrm{m}_{1}, \mathrm{~m}_{2}, \ldots . \mathrm{m}_{\mathrm{n}-1}\right)$ means the $\mathrm{nth}$ order crossculmulants between the reference signal and filter out Then,

$C_{x z \ldots z}\left(m_{1}, m_{2}, \ldots, m_{n-1}\right.$

$=\sum \mathrm{g}(\mathrm{j}) C_{z z \ldots z}\left(j+m_{1}, j+m_{2}, \ldots, j+m_{n-1}\right.$

$C_{y z \ldots z}\left(m_{1}, m_{2}, \ldots, m_{n-1}\right.$

$$
=\sum_{j=0}^{N-1} \mathrm{~h}(\mathrm{j}) C_{z z \ldots z}\left(j+m_{1}, j+m_{2}, \ldots, j+m_{n-1}\right.
$$

The error is expressed as

$\xi=\sum \quad \sum_{m 1} \ldots \sum_{m_{n-1}}\left[C_{x z_{x y z}}\left(m_{1}, m_{2}, \ldots, m_{n-1}\right)-\right.$ $\left.C_{y z . z}\left(m_{1}, m_{2}, \ldots, m_{n-1}\right)\right]^{2}$

The gradient algorithm is used to update the filter coefficients. Its convergence rate is quicker compared to SOS-based filter, and it is very insensitive to Gaussian noise, but its output is sometimes not very stable. Thus, base filtering of genetic information is adapted.The search for the optimum solution is based on the concept of natural selection. It consists of three major activities: aberrance, inheritance, and cross-breed. The excellent solution is kept through the above three activities and the bad one is discarded.The chromosome's original state is not very essential because the genetic algorithm can check the entire room. We can define the algorithm as follows:

(1) Coding of Chromosome

(2) Computing the aim function.

(3) Performing the correlation operations.

Simulation:

In our simulation the MA model interference is measured and we used the fourth-order cumulant.The assessment of fourth-order cumulant is expressed as

$\hat{\mathrm{C}} \hat{\mathrm{C}}_{x z z}\left(q, m_{1}, m_{2}, m_{3}\right)=\hat{\mathrm{R}}_{x z z}\left(q, m_{1}, m_{2}, m_{3}\right)-$

$\hat{\mathrm{R}}_{x z}\left(q, 0, m_{1}\right) \hat{\mathrm{R}}_{x z}\left(q, m_{2}, m_{3}\right)-$

$\hat{\mathrm{R}}_{x z}\left(q, 0, m_{2}\right) \hat{\mathrm{R}}_{x z}\left(q, m_{1}, m_{3}\right)-$

$\hat{\mathrm{R}}_{x z}\left(k, 0, m_{3}\right) \hat{\mathrm{R}}_{x z}\left(k, m_{1}, m_{2}\right)$

Where

$$
\begin{aligned}
\hat{\mathrm{R}}_{x z z z}\left(q, m_{1}, m_{2}, m_{3}\right) & \\
& =\frac{1}{q} \sum_{i=1}^{q} x(i) z\left(i+m_{1}\right) z\left(i+m_{2}\right) z(i \\
& \left.+m_{3}\right)
\end{aligned}
$$

$\hat{\mathrm{R}}_{x z}\left(\mathrm{q} ; l_{1}, l_{2}\right)=\frac{1}{q} \sum_{i=1}^{q} x\left(i+l_{1}\right) z\left(i+l_{2}\right)$

Simulation parameters:

The training of filter coefficients is done using two methods:

1. gradient algorithm

2. genetic algorithm

The signal of concern in computer simulation is a DS signal, the reference signal is produced by a sine wave excited MA scheme, the MA parameter is given as [ $1 ;-0.2,0.2,0.7 ;-0.65,0.3 ;-0.251]$.The JSR is $20 \mathrm{~dB}$, the adaptive filter order is 16 , and randomly chosen its original state.

Frequency selection:

It is selection by the Pseudo noise which is sent by the sender to the receiver. Based on the PN code filter frequency is selected.

Filter algorithm:

Spread spectrum:

Transmitter (M, S)

$\mathrm{P} \leftarrow \mathrm{M}$

For $i=1, \ldots \ldots \ldots \ldots$ do

$$
\begin{aligned}
& \mathrm{S}_{\mathrm{i}}[\mathrm{i} \ldots \ldots \ldots . \mathrm{S}] \leftarrow \mathrm{S}[\mathrm{i} \ldots \ldots \ldots . . \mathrm{S}] \\
& \mathrm{S}_{\mathrm{i}}[1 \ldots \ldots . \mathrm{i}-1] \leftarrow \mathrm{C}[1 \ldots \ldots \ldots \mathrm{i}-1] \\
& \mathrm{M}_{\mathrm{i}} \leftarrow \mathrm{P}_{\mathrm{i}}\left[1 \ldots \ldots \ldots . \mathrm{P}_{\mathrm{i}} / 2\right] \\
& \mathrm{PN}_{\mathrm{i}} \leftarrow \mathrm{PN}\left(\mathrm{S}_{\mathrm{i}}\right)
\end{aligned}
$$

Published By:

Blue Eyes Intelligence Engineering 
Spread $\mathrm{M}_{\mathrm{i}}$ with $\mathrm{PN}_{\mathrm{i}}$

$$
\mathrm{P}_{\mathrm{i}+1} \leftarrow \mathrm{P}_{\mathrm{i}}\left[\left|\mathrm{M}_{\mathrm{i}}\right|+1, \ldots \ldots .\left|\mathrm{P}_{\mathrm{i}}\right|\right]
$$

End for

Where :

$\mathrm{M}$ - Message

$\mathrm{S}$ - Secret key

$\mathrm{P}$ - left message

PN - Pseudo Noise

Receiver :

RECEIVER(S, R, n, l)

OldCache \$ GetCache (S)

a \$ MACAddress (R)

for all CurrentBuffer $\$$ GetBuffer (S) do

Corr $[1, \ldots$, nl $]$ \$ FastCorrelate (CurrentBuffer, a)

for all $\mathrm{j} !\{1, \ldots, \mathrm{nl}\}$ do

if Corr $[j]>$ threshold then

end if push $\mathrm{j}$ into $\mathrm{PEoM}[]$

end for

\section{Empty Cache:}

For

if PEoM[] is empty then

OldCache \$ CurrentCache

else

Cache \$ concat (OldCache, CurrentCache)

KeyInfer (Cache, PEoM)

end if

end for

end procedure

Where:

PEoM - Possible end of message

KeyInfer - Infer key function

S - Sampled data

l - length of message

$\mathrm{R}$ - rate of transfer

$\mathrm{n}$ - number of bits in key

Parameter used for simulation:

\begin{tabular}{|c|c|}
\hline Spread Spectrum factor & $\mathbf{1 0 0}$ \\
\hline Packet size & 1024 bits \\
\hline Size of key & 10 \\
\hline Jammer signal range & $-30 \mathrm{~dB}$ to $20 \mathrm{~dB}$ \\
\hline Frequency & $2.4 \mathrm{GHz}$ \\
\hline Modulation & BPSK \\
\hline Noise power & $-20 \mathrm{~dB}$ \\
\hline
\end{tabular}

IV. RESULTS

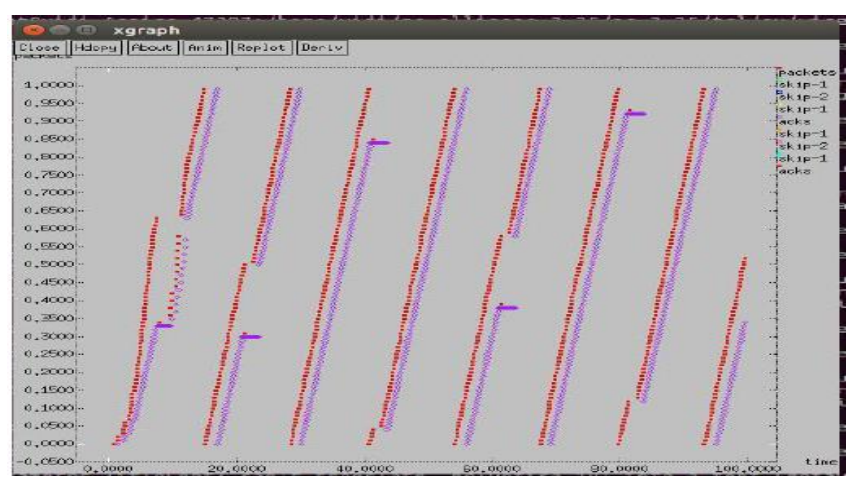

Fig 1 : Spectrum frequencies after filtering
The figure above shows the appropriate frequency spectrum of the filter after application. The figure above shows after application and after application of the filter. The energy corresponding to the $50 \mathrm{~Hz}$ is $-26.29 \mathrm{~dB}$ from the frequency spectrum for the pre-filtration. When the notch filter is applied, the energy is decreased to $-35.75 \mathrm{~dB}$ by the $50 \mathrm{~Hz}$ signal. It shows that the filter decreases the interference of the power line in the signal.

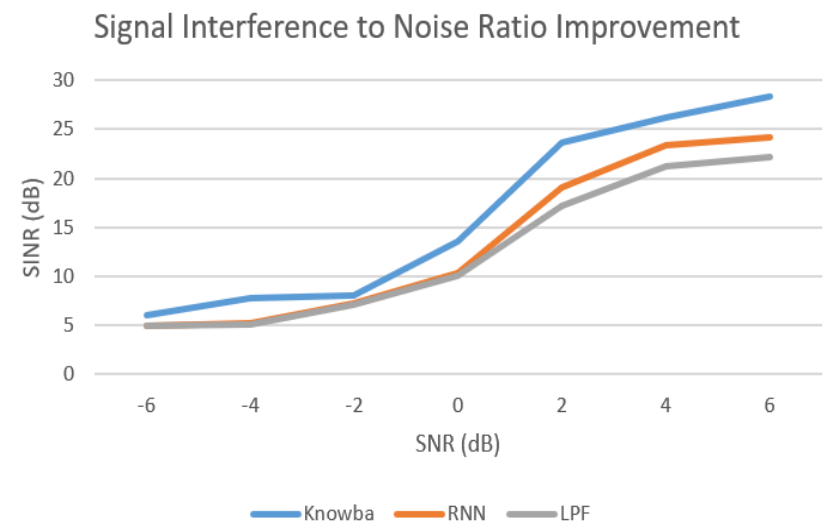

Fig. 2 Signal Interference to Noise Ratio improvement

Above figure shows the Improvement over the signal interference to noise ratio vs Signal Noise ratio. We compared the proposed Knowba results with the Recurrent Neural Network based (RNN) predictor and Liner predictor based low pass filter (LPF).

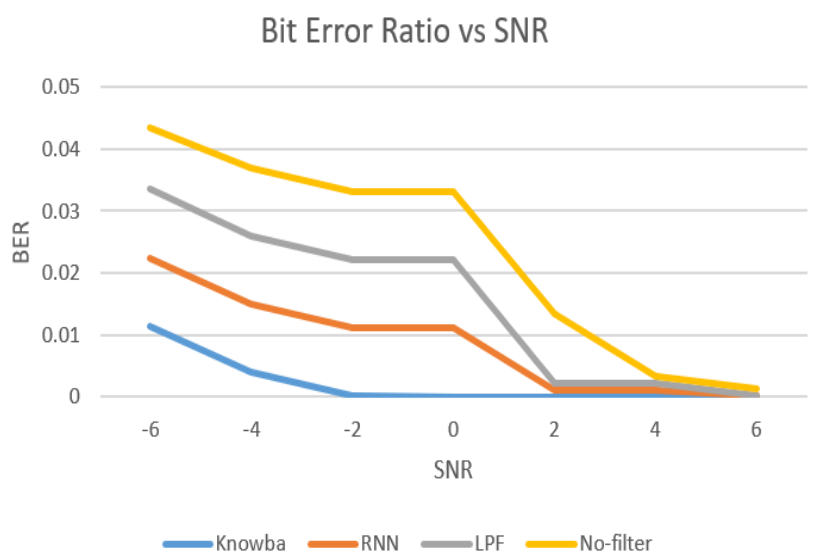

Fig 3 Bit Error Ratio vs SNR

Above figure shows the Improvement over the Bit Error Ratio vs Signal to Noise ratio (dB). We compared the proposed Knowba results with the Recurrent Neural Network based (RNN) predictor and Liner predictor based low pass filter (LPF).

\section{CONCLUSIONS}

In this paper we propose a new knowledge based strategy to reject interference in narrowband Direct sequence spread spectrum system. It is shown that this system achieves significantly better SINR and BER performance than system based on Recurrent neural network and low pass filter. The energy is decreased to $-35.75 \mathrm{~dB}$ by the $50 \mathrm{~Hz}$ signal when filter is used. 


\section{Implementation of Knowba Filtering Method for Intereference Rejection in NBDSSS}

\section{REFERENCES}

1. Z. X. Ma, M. Zhang, S. Shaham, S. P. Dang, and J. Hart, "Literature review of the communication technology and signal processing methodology based on the smart grid," Applied Mechanics and Materials, vol. 719, pp. 436-442, 2015.

2. I. Rpke, T. H. Christensen, and J. O. Jensen, "Information and communication technologies a new round of household electrification," Energy Policy, vol. 38, no. 4, pp. 1764 - 1773, 2010.

3. Y. Park, J. Ha, S. Kuk, H. Kim, C. J. Liang, and J. Ko, “A feasibility study and development framework design for realizing smartphone based vehicular networking systems," IEEE Transactions on Mobile Computing, vol. 13, no. 11, pp. 2431-2444, Nov. 2014.

4. C. C. Huang, P. Y. Lee, and P. Y. Chen, "Implementation of a smartphone based portable doppler flowmeter," in Proc. IEEE International Ultrasonics Symposium, Oct. 2011, pp. 1056-1059.

5. A. Rahmati and L. Zhong, "Studying smartphone usage: Lessons from a four-month field study," IEEE Transactions on Mobile Computing, vol. 12, no. 7, pp. 1417-1427, July 2013.

6. M. Sauter, 3G, 4G and Beyond: Bringing Networks, Devices and the Web Together, Wiley, 2012.

7. J. Proakis and M. Salehi, Digital Communications, McGraw-Hill Education, 2007.

8. S. Dang, J. P. Coon, and D. Simmons, "Combined bulk/per-tone relay selection in two-hop OFDM systems," Wireless

9. Communications Letters, IEEE, 2015 (under review).

10. Z. Ma, A. Gholamzadeh, B. Tang, S. Dang, and S. Yang, "Matlab based simulation of the efficiency of the complex ofdm on power line communication technology," in Proc. Fourth International Conference on Instrumentation and Measurement, Computer, Communication and Control, Sept. 2014, pp. 374-378.

11. S. Dang, J. P. Coon, and D. Simmons, "Combined bulk and pertone relay selection in super dense wireless networks," in Proc. IEEE ICC, London, United Kingdom, Jun. 2015.

12. Q. Ding, A Story of Wireless Communication, Posts and Telecom Press, 2010.

13. P. Baier, "A critical review of CDMA," in Proc. IEEE 46th Vehicular Technology Conference, Apr. 1996, vol. 1, pp. 6-10.

14. H. V. Poor and L. A. Rusch, "Narrowband interference suppression in spread spectrum cdma," IEEE Personal

15. Communications Magazine, vol. 1, no. 3, pp. 14-27, 1994.

16. T. Yucek and H. Arslan, "A survey of spectrum sensing algorithms for cognitive radio applications," Communications Surveys Tutorials, vol. 11, no. 1, pp. 116-130, First 2009.

17. D. Koulakiotis and A. Aghvami, "Data detection techniques for ds/cdma mobile systems: A review," Personal Communications, vol. 7, no. 3, pp. 24-34, Jun. 2000.

18. J. Kim, A. Marathe, G. Pei, S. Saha, B. Subbiah, and A. Vullikanti, "Analysis of policy instruments for enhanced competition in spectrum auction," in Proc. IEEE International Symposium on Dynamic Spectrum Access Networks, Oct. 2012, pp. 89-96.

19. S. Gandhi, C. Buragohain, L. Cao, H. Zheng, and S. Suri, "A general framework for wireless spectrum auctions," in Proc. 2nd IEEE International Symposium on New Frontiers in Dynamic Spectrum Access Networks, April 2007, pp. 22-33.

20. S. Greenstein, "The revolution in spectrum allocation," IEEE Micro, vol. 29, no. 3, pp. 4-6, May 2009.

21. X. Geng and A. Whinston, "Profiting from value-added wireless services," Computer, vol. 34, no. 8, pp. 87-89, Aug. 2001.

22. S. Tabrizi, M. Miller, and J. Lee, "Cdma cellular system capacity in the military environment," in Proc. MILCOM 97, Nov. 1997, pp. 1163-1167.

23. K. Bhargav and R. Singhal, "Zigbee based vanets for accident rescue missions in 3G WCDMA networks," in Proc. IEEE Global Humanitarian Technology Conference: South Asia Satellite, Aug. 2013, pp. 310-313.

24. D. Jang, S. Choi, and T. Park, "Development of collapse-sensing phone for emergency positioning system," in Proc. Sixth International Conference on Information Technology: New Generations, April 2009, pp. 1649-1652.

25. X. Duan, Z. Niu, and J. Zheng, "Downlink transmit power minimization in power-controlled multimedia CDMA systems," in Proc. 13th IEEE International Symposium on Personal, Indoor and Mobile Radio Communications, Sept. 2002, pp. 1102-1106.

26. A. Denk, "Detection and jamming low probability of intercept (LPI) radars," Ph.D. dissertation, Monterey California. Naval Postgraduate School, 2006.

27. D. So, Lecture Notes of Digital Mobile Communications, The University of Manchester, 2014. Workshops, Nov. 2004, pp. 120-129. 1782-1785. IEEE, 2000. IEEE, 2016
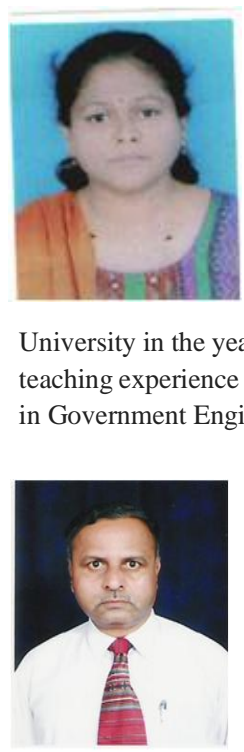
Bellur cross, Mandya District,Karnataka,India.

29. Taijie, Li, Hu Guangrui, and Gu Qing. "Interference rejection in direct-sequence spread spectrum communication systems based on higher-order statistics and genetic algorithm." In WCC 2000-ICSP 2000. 2000 5th International Conference on Signal Processing Proceedings. 16th World Computer Congress 2000, vol. 3, pp.

30. Tang, Zheng, Feng Zhou, and Wenting Zheng. "Pulse position modulation spread spectrum underwater acoustic communication system using NH sequence." In 2016 IEEE International Conference on Signal Processing, Communications and Computing (ICSPCC), pp. 1-4.

\section{AUTHOR DETAILS}

Mrs Rekha R S is aresearch scholar perceiving her Ph.D in PET Research centre(PESCE), Mandya, under VTU Part Time Ph.D Programme from 2016 in wireless communication system. Her area of research interests are wireless communication system, spread spectrum communication system, Digital signal Processing, etc. She perceived Bachelor of Engineering degree in Electronics and Communication Engineering degree from Karnatak University in the year1998 and M.Tech from VTU in the year 2008. She has teaching experience of 15 years and presently working as Assistant Professor in Government Engineering College, Chamarajanagara.

Dr. K.N Muralidhara completed his B.E. in Electronics and Communication Engineering from University of Mysore in 1981, M.E. in 1990 and Ph.D in 1998,both from Indian Institute of technology,Roorkee,India. He was Professor and head of E \& C engineering department in PES college of engineering,Mandya, Karnataka,India. He has guided many Ph.D.students in the areas of VLSI,Mobile Communication,Wireless Communication, computer simulation. At present, he is working as Professor in E \& C engineering department in Adichunchanagiri University, Balagangadharanath nagar, 